Swarthmore College

Works

Physics \& Astronomy Faculty Works

Physics \& Astronomy

3-1-2011

\title{
Calibrated Cylindrical Mach Probe In A Plasma Wind Tunnel
}

Xingyu Zhang , '12

Daniel R. Dandurand , '11

Timothy G. Gray , '01

Michael R. Brown

Swarthmore College, doc@swarthmore.edu

Vyacheslav S. Lukin , '00

Follow this and additional works at: https://works.swarthmore.edu/fac-physics

Part of the Physics Commons

Let us know how access to these works benefits you

\section{Recommended Citation}

Xingyu Zhang , '12; Daniel R. Dandurand, '11; Timothy G. Gray , '01; Michael R. Brown; and Vyacheslav S. Lukin , '00. (2011). "Calibrated Cylindrical Mach Probe In A Plasma Wind Tunnel". Review Of Scientific Instruments. Volume 82, Issue 3. DOI: 10.1063/1.3559550

https://works.swarthmore.edu/fac-physics/98

This work is brought to you for free by Swarthmore College Libraries' Works. It has been accepted for inclusion in Physics \& Astronomy Faculty Works by an authorized administrator of Works. For more information, please contact myworks@swarthmore.edu. 


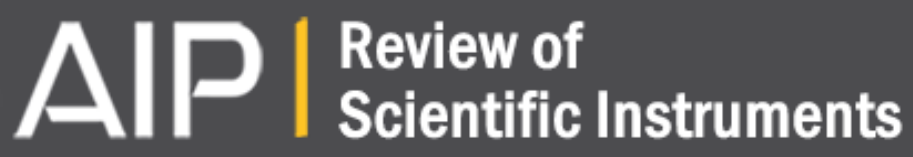

\section{Calibrated cylindrical Mach probe in a plasma wind tunnel}

X. Zhang, D. Dandurand, T. Gray, M. R. Brown, and V. S. Lukin

Citation: Review of Scientific Instruments 82, 033510 (2011); doi: 10.1063/1.3559550

View online: http://dx.doi.org/10.1063/1.3559550

View Table of Contents: http://scitation.aip.org/content/aip/journal/rsi/82/3?ver=pdfcov

Published by the AIP Publishing

\section{Articles you may be interested in}

Diode calibration of a Langmuir probe system for measurement of electron energy distribution functions in a plasma

Rev. Sci. Instrum. 76, 086105 (2005); 10.1063/1.2006287

Probe characterization of high-current driven metal plasma in a vacuum-arc rail gun

J. Appl. Phys. 96, 4084 (2004); 10.1063/1.1786344

Response to "The invalidity of a Mach probe model" [Phys. Plasmas 9, 1832 (2002)]

Phys. Plasmas 9, 1837 (2002); 10.1063/1.1464889

Response to "The invalidity of a Mach probe model" [Phys. Plasmas 9, 1832 (2002)]

Phys. Plasmas 9, 1835 (2002); 10.1063/1.1464888

Response to "The invalidity of a Mach probe model" [Phys. Plasmas 9, 1832 (2002)]

Phys. Plasmas 9, 1834 (2002); 10.1063/1.1464887

\section{ZABER}

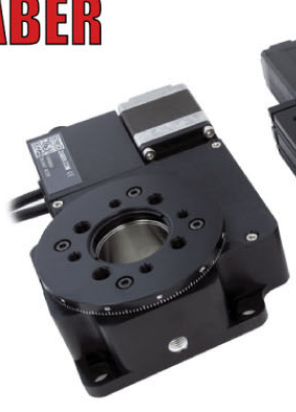

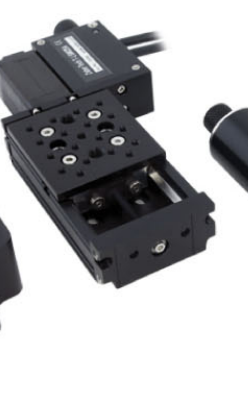

Automate your research applications with Zaber's line of high precision positioning devices.

Low cost. Built-in controllers. Simple to set up and easy to use.

Learn more at zaber.com 


\title{
Calibrated cylindrical Mach probe in a plasma wind tunnel
}

\author{
X. Zhang, ${ }^{1, \text { a) }}$ D. Dandurand, ${ }^{1}$ T. Gray, ${ }^{1}$ M. R. Brown, ${ }^{1}$ and V. S. Lukin ${ }^{2}$ \\ ${ }^{1}$ Department of Physics and Astronomy, Center for Magnetic Self Organization, Swarthmore College, \\ Swarthmore, Pennsylvania 19081-1397, USA \\ ${ }^{2}$ Space Science Division, Naval Research Laboratory, Washington, DC 20375, USA
}

(Received 12 October 2010; accepted 6 February 2011; published online 24 March 2011)

\begin{abstract}
A simple cylindrical Mach probe is described along with an independent calibration procedure in a magnetized plasma wind tunnel. A particle orbit calculation corroborates our model. The probe operates in the weakly magnetized regime in which probe dimension and ion orbit are of the same scale. Analytical and simulation models are favorably compared with experimental calibration. (C) 2011 American Institute of Physics. [doi:10.1063/1.3559550]
\end{abstract}

\section{INTRODUCTION}

A Mach probe is a directional Langmuir probe used to measure local plasma flow. Fundamentally, a Mach probe operates by collecting ion flux from opposite directions. If there is no net flow $v_{d}$ and the Mach number $M \equiv v_{d} / c_{s}=0$ (where $c_{s}$ is the sound speed), then the ratio of upstream to downstream ion flux should be unity. A net flow in the upstream direction will increase the ratio. The classic 1D model for Mach probe analysis due to Hudis and Lidsky ${ }^{1}$ relates upstream and downstream ion collection currents in an unmagnetized plasma to the Mach number $M: I_{\text {up }} / I_{\text {down }}$ $=\exp (K M)$ with the constant $K$ of order unity for comparable electron and ion temperatures $T_{e} \sim T_{i}$. Criticism of the Hudis-Lidsky model ${ }^{2}$ challenges its assumptions; for example, physical probes impede the downstream flow so the model needs to be at least 2D. Nonetheless, other researchers find good experimental correspondence with the HudisLidsky form $I_{\text {up }} / I_{\text {down }}=\exp (K M)$ with varying values of the constant $K^{3-6}$

We present here discussion of a simple "Gundestruptype" Mach probe ${ }^{5,7,8}$ used in the Swarthmore Spheromak Experiment (SSX). ${ }^{9}$ An accurate calibration method based on magnetic time-of-flight in a magnetized wind tunnel is presented and a particle orbit calculation is performed. We operate in the regime where probe dimension and ion orbit are of the same scale $(\sim 1 \mathrm{~cm})$ so our experiments are in neither the magnetized nor unmagnetized limit. In addition, we operate in a regime where $T_{i} \geq T_{e}$, different from those of other researchers.

In Sec. II, the theoretical underpinnings of Mach probe operation are reviewed. In Sec. III, the experimental setup, Mach probe design, calibration, and results are presented. Comparison with a particle orbit simulation is presented in Sec. IV. Discussion and a summary are presented in Sec. V.

\section{THEORETICAL MOTIVATION}

The fundamental equation for modeling the Mach probe is:

$$
\frac{J_{\text {up }}}{J_{\text {down }}}=e^{K M},
$$

a)Electronic mail: xzhang9@swarthmore.edu. where $J_{\text {up }}$ is the current density measured by the upstream probe and $J_{\text {down }}$ is the current density measured by the downstream probe, and $K$ is a calibration constant typically of order unity. ${ }^{10}$ An argument for this form is presented below. $M$ is the Mach number:

$$
M=\frac{v_{d}}{c_{s}},
$$

where $v_{d}$ is the ion drift velocity and $c_{s}$ is the sound speed. The sound speed, $c_{s}$, is defined:

$$
c_{s}=\gamma \sqrt{\frac{T_{e}+T_{i}}{m_{i}}},
$$

where $\gamma$ is the ratio of specific heats in the plasma (taken to be unity here), $T_{e}$, and $T_{i}$ are the electron and ion temperatures, respectively, and $m_{i}$ is the ion mass (protons here). Boltzmann's constant has been absorbed into $T_{i}$ and $T_{e}$ in Eq. (3).

The form for the ratio of upstream and downstream currents in Eq. (1) can be determined using fluid ion theory. ${ }^{1,10}$ Even in the absence of a detailed physical model, however, an exponential form for the current ratio can be seen as a simple consequence of the properties of a shifted 1D Maxwellian velocity distribution. The following calculation was published by Mott-Smith and Langmuir ${ }^{11}$ and recently by Oksuz. ${ }^{12} \mathrm{We}$ reproduce the analysis here.

The probability distribution for a shifted Maxwellian velocity distribution is given by

$$
f(v)=\sqrt{\frac{m_{i}}{2 \pi E_{0}}} e^{-m_{i}\left(v-v_{d}\right)^{2} / 2 E_{0}},
$$

where $E_{0}=k T_{i}$, with $k$ Boltzmann's constant. The net upstream and downstream current densities are then given by, respectively,

$$
\begin{aligned}
J_{\text {up }} & =\int_{0}^{\infty} n_{0} q v f(v) d v \\
J_{\text {down }} & =\int_{-\infty}^{0} n_{0} q v f(v) d v,
\end{aligned}
$$




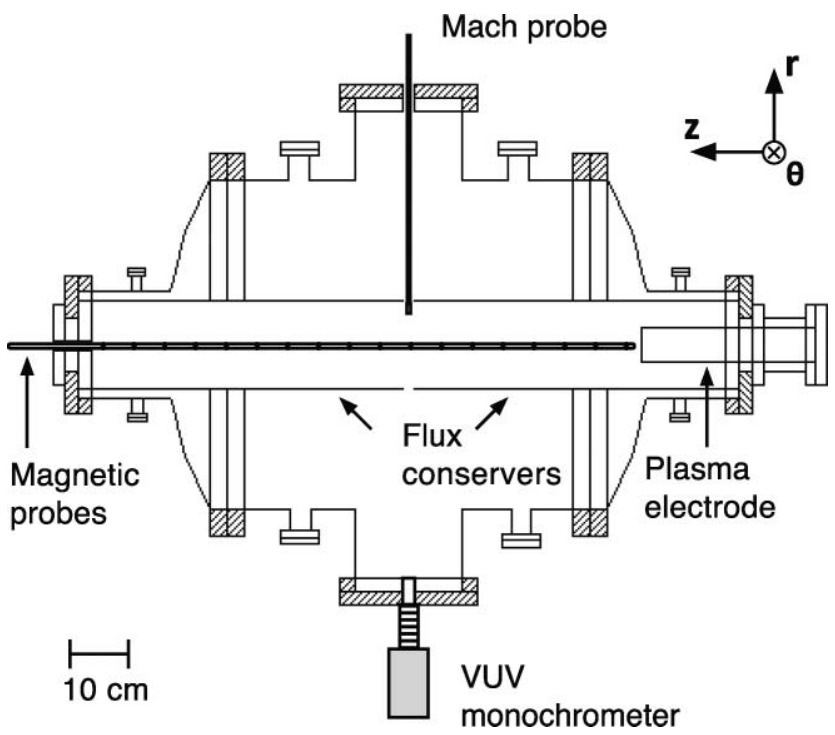

FIG. 1. Mach probe and magnetic probe setup inside the SSX plasma wind tunnel. The dimensions of the tunnel are $R=0.08 \mathrm{~m}, L=1.0 \mathrm{~m}$. Device coordinates are as indicated with the $\theta$-direction pointing into the page and the $z$ along the magnetic probe. The $r$-direction is radially out from the axis. The plasma originates from the electrode and flows in the positive $z$-direction (from right to left in the figure).

where $q$ is the ion charge and $n_{0}$ is the ion density. Taking the absolute value of the ratio of the currents, we get

$$
\frac{J_{\text {up }}}{J_{\text {down }}}=\frac{e^{-r^{2}}+r \sqrt{\pi}(1+\operatorname{erf}(r))}{e^{-r^{2}}-r \sqrt{\pi}(1-\operatorname{erf}(r))},
$$

where $r$ is defined to be $v_{d} / v_{t h}$, with $v_{t h}=\sqrt{2 E_{0} / m_{i}}$. The above function is well approximated by $e^{K M}$ for $M$ order unity and $K$ a constant of order unity. As an example, for Mach numbers up to 1.5 , the maximum error obtained using this approximation is $6 \%$.

Once we have determined the relationship between collected current and Mach number [Eq. (1)], it remains to determine the constant $K$. We have seen that $K$ is of order unity but unfortunately, there is very little analytical guidance available to determine $K$ for our case: $T_{i} / T_{e} \geq 1, r_{\text {probe }} / \rho_{i} \sim 1$, and cylindrical probe geometry. We have applied the various models surveyed in Hutchinson ${ }^{13}$ using the SSX parameters.

The Hudis/Lidsky model $^{1}$ has a prediction of $K$ $=4 \sqrt{T_{e} T_{i}} /\left(T_{e}+T_{i}\right)=1.73$ using SSX wind tunnel temperatures discussed below $\left(T_{e}=7 \mathrm{eV}, T_{i}=21 \mathrm{eV}\right)$. However, the Hudis/Lidsky model was derived for $T_{i} \ll T_{e}$. In addition, the model is one-dimensional and unmagnetized.

Hutchinson $^{13}$ performed a Mach probe calculation and simulation for a range of temperatures and magnetizations. In particular, for $T_{i} \leq 3 T_{e}$ in the weakly magnetized case, a nearly universal constant $K=1.34$ is found. In the same paper, a "free-flight" formula for unmagnetized plasma $K$ $=\sqrt{2 \pi T_{e} / T_{i}}=1.45$ for the SSX wind tunnel case is calculated. These formulas were calculated for spherical geometry and may not apply in our cylindrical case. Hutchinson also reports a formula due to Schats $K=4 \sqrt{T_{e} / T_{i}}=2.3$ for the unmagnetized case.

Recently, Patacchini and Hutchinson have expanded upon Hutchinson's earlier numerical simulations to pro-

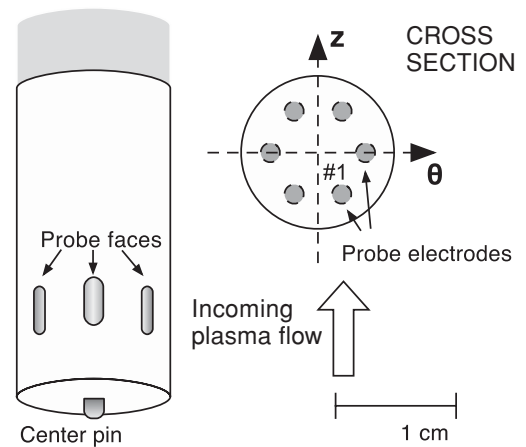

FIG. 2. Close-up of Mach probe showing orientation of the electrode collector areas and device coordinates. Probes are numbered clock-wise beginning bottom-right (e.g., probes 3 and 6 form the $\theta$ pair).

vide three-dimensional calibration models for more realistic geometries across a range of magnetization parameters. ${ }^{14}$ However, more studies in the weakly magnetized regime are needed to experimentally verify these results and to provide parameters for Mach probe calibration for probes with realistic geometries.

\section{SSX MACH PROBE AND WIND TUNNEL}

The Swarthmore Spheromak Experiment (SSX; Ref. 9) is a flexible facility used to study plasma merging and magnetic reconnection. The SSX device features a $L \cong 1 \mathrm{~m}$ long, high vacuum chamber in which we generate $n \geq 10^{20} \mathrm{~m}^{-3}, T$ $\geq 20 \mathrm{eV}, B \cong 0.1 \mathrm{~T}$ hydrogen plasmas. Plasma plumes are generated by pulsed magnetized plasma guns at either end of the device. Plasmas are accelerated to high velocity ( $\leq 100 \mathrm{~km} / \mathrm{s}$ ) by the discharge current in the guns $(\leq 100 \mathrm{kA})$ and injected into a highly evacuated target volume called a flux conserver. The flux conserver is usually cylindrical in shape and bounded by a thick, highly conducting copper shell. In a typical experiment, plasma plumes are injected at either end of a flux conserver and dynamical merging and relaxation ensues. $^{15}$

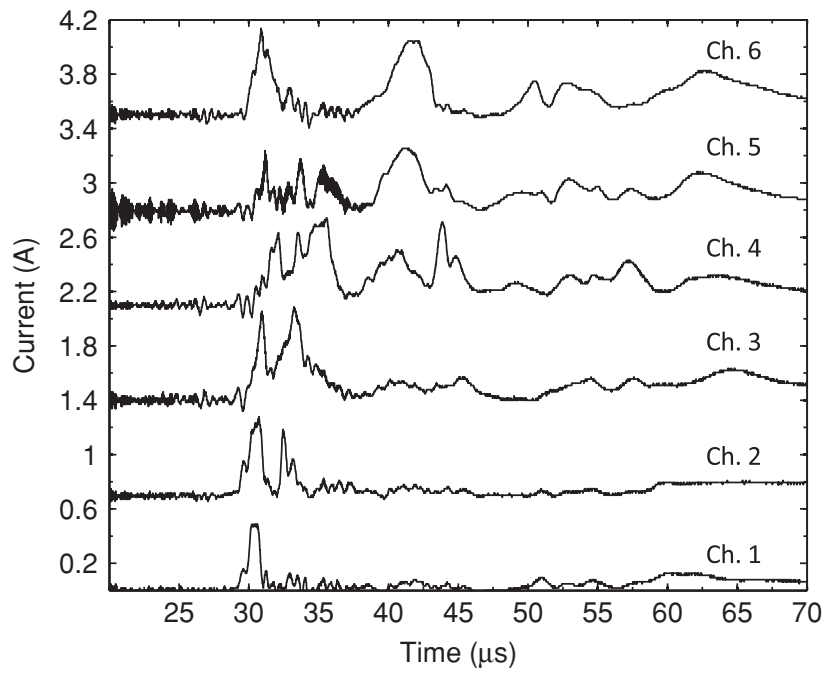

FIG. 3. Typical current measurements for each of the six electrode collectors of the Mach probe with channels labeled. The currents from each probe face have been offset by 0.7 A for clarity. 


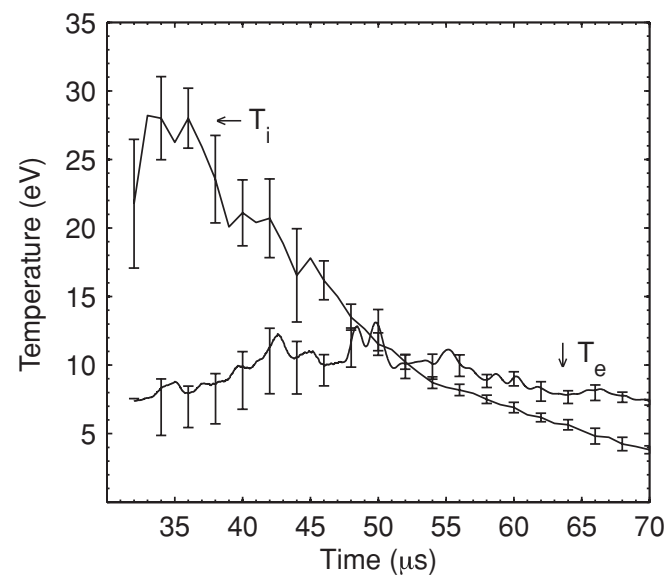

FIG. 4. $T_{e}$ was determined using VUV spectroscopy and $T_{i}$ with the IDS Note that $T_{e}$ remained below $15 \mathrm{eV}$ for the duration of the discharge and that $T_{i}>T_{e}$ early in time.

For this study, we have implemented a single plasma source in a high aspect ratio "wind tunnel" configuration (see Fig. 1). The wind tunnel has dimensions $R=0.08 \mathrm{~m}$ and $L=1.0 \mathrm{~m}$. The plasma gun can inject a magnetized plasma plume of either right-handed (RH) or left-handed (LH) magnetic helicity. Operationally, this means that the discharge current in the gun can be either aligned or antialigned with the magnetic field imbedded in the inner electrode (referred to as "stuffing flux," $\Phi_{\text {gun }}$, in prior work). The magnetic helicity of the plume also determines the helical pitch of magnetic field lines in the final relaxed state in the wind tunnel. ${ }^{16}$

A cryogenic pump provides the high vacuum $\left(10^{-8}\right.$ torr) for the plasma wind tunnel. The interior walls of the wind tunnel are maintained by helium glow discharge conditioning (GDC; $0.1 \mathrm{~A}, 400 \mathrm{~V}, 50 \mu \mathrm{m}$ of $\mathrm{He}$ ) and baking with a thermal blanket $\left(100{ }^{\circ} \mathrm{C}\right)$ for several hours. In order to clean the Mach probe, collector faces are biased at $-60 \mathrm{~V}$ during $\mathrm{He}$ glow to collect ion current at the probe faces. We find that the plasma wind tunnel and probe need to be glowed and baked again after 30-60 discharges for best results.

All discharges in this study had identical external parameters: $I_{\text {gun }}=80 \mathrm{kA}, \Phi_{\text {gun }}=1.0 \mathrm{mWb}, W_{\text {bank }}=5.0 \mathrm{~kJ}$. We found that a probe bias of $-20 \mathrm{~V}$ was sufficient to collect ion saturation current without prohibitive arcing. Since $T_{e}$ $\sim 10 \mathrm{eV}$, this bias is more than two electron temperatures below the plasma potential. A scan of the Mach probe bias voltage confirmed that electron flux was satisfactorily repelled at $-20 \mathrm{~V}$.

The SSX Mach probe has a cylindrical Gundestrup geometry in which six evenly spaced tungsten electrodes are encased in a boron nitride turret (see Fig. 2). The ceramic turret is $2.54 \mathrm{~cm}$ in length and $1.3 \mathrm{~cm}$ in diameter. The electrode collector faces are recessed into the ceramic housing by $2.0 \mathrm{~mm}$. Each rectangular opening has length $4.0 \mathrm{~mm}$, width $1.6 \mathrm{~mm}$, and area $A=6.0 \pm 0.1 \mathrm{~mm}^{2}$. Individual electrode collectors effectively provide independent current measurements when biased with respect to the central pin, which protrudes from the end of the turret $1.6 \mathrm{~mm}$. The exposed electrode collector areas are the same within $2 \%$. The probe is mounted at

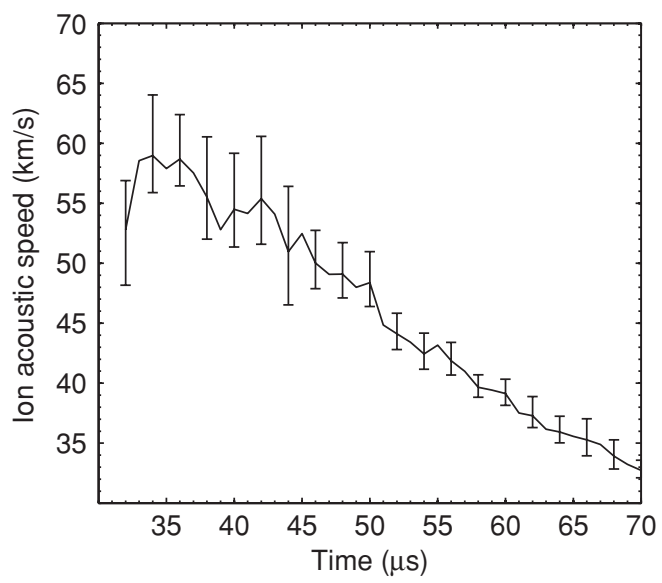

FIG. 5. Using Eq. (3), the sound speed is calculated as a function of time.

the midplane of the SSX vessel, and inserted $5.5 \mathrm{~cm}$ from the center of the flux conserver for these calibration studies. The probe is otherwise moveable in the radial direction. The Mach probe is oriented with two adjacent faces facing the initial plasma flow and two opposing faces aligned in the cross-flow $(\theta)$-direction as depicted in Fig. 2.

Each collector is biased using a separate $180 \mu \mathrm{F}$ capacitor bank charged with an external power supply that is isolated during the plasma discharge to prevent ground loops. The voltage droop is typically less than $1 \%$ during a discharge so the voltage at each collector face is fixed. Six matched, high bandwidth $(20 \mathrm{MHz})$ current transformers (Pearson model 411), one for each collector face, report the ion current from each probe face. Typical ion current magnitudes were $\leq 1 \mathrm{~A}$ consistent with $I=n e v_{\text {th }}$ A.

As shown in Fig. 2 and using Eq. (1), we measure the local Mach number resolved into the directions of the three probe face pairs: one pair $M_{1}$ at $-30^{\circ}$ with respect

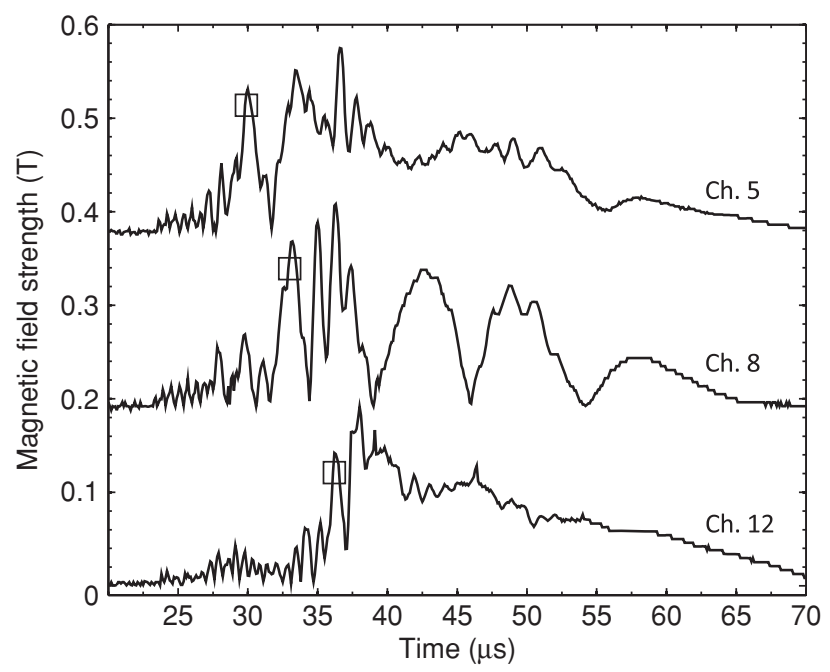

FIG. 6. $|\mathbf{B}|$ as a function of time for a single discharge. Measurements are displayed for probes 5, 8, and 12 of the linear array, 24.2, 38.6, and $58.0 \mathrm{~cm}$ from the first magnetic probe, respectively, beginning with the top trace. Squares indicate the time of detection the leading edge as the plasma approaches each probe. The magnetic field strengths have been offset for clarity. 


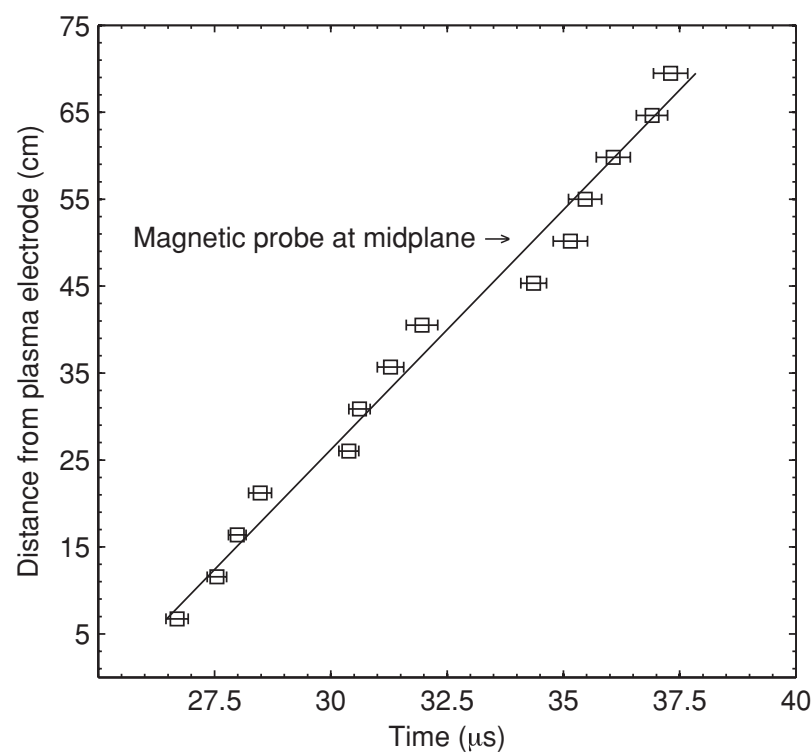

FIG. 7. The initial plume velocity, $v_{\text {plume }}$, determined using a linear array of magnetic probes. Using the slope of a linear regression of the mean arrival times (squares) for each probe, $v_{d}=55.1 \pm 6.4 \mathrm{~km} / \mathrm{s}$ for data aggregated over 40 discharges.

to the initial flow direction, another pair $M_{2}$ at $+30^{\circ}$, and the last pair $M_{3}$ at $90^{\circ}$ (to measure the $\theta$-direction or crossflow). The rotation orientation of the probe is arbitrary but we chose this convention for convenience and also to prevent a probe collector from having a direct line-of-sight to the plasma gun. Any other rotation orientation of the probe could be used and resolved in the probe's symmetry axis. Flow vectors can then be projected onto the machine axes.

For an arbitrary flow vector $\mathbf{F}$ with components $F_{z}$ and $F_{\theta}$, the first probe direction measures $M_{1}=F_{z} \cos 30^{\circ}-F_{\theta} \sin 30^{\circ}$, the second measures $M_{2}$ $=F_{z} \cos 30^{\circ}+F_{\theta} \sin 30^{\circ}$, while the third measures $M_{3}$ $=F_{\theta}$. Assuming that the signal is insensitive to the direction of the magnetic field at the probe, the system is over-determined and we only have information in the $\hat{z}$ - and $\hat{\theta}$-directions. We can solve for the components of F: $F_{z}=\left(M_{1}+M_{2}\right) / 2 \cos 30^{\circ}$ and $F_{\theta}=\left(M_{2}-M_{1}\right) / 2 \sin 30^{\circ}$. We have additional information about $F_{\theta}$ from $M_{3}$ so we choose to construct the average: $F_{\theta}=\left[M_{3}+\left(M_{2}-M_{1}\right) / 2 \sin 30^{\circ}\right] / 2$.

In order to corroborate our analysis, the probe was also rotated by $15^{\circ}, 30^{\circ}$, and $180^{\circ}$ from the orientation described above, which gave the same initial flow speeds. This procedure is typical for confirming that the probe operated under a weakly magnetized regime. ${ }^{5}$

\section{A. Data}

In Fig. 3, we show typical ion current measurements for the six electrode collectors of the Mach probe on a single discharge. The plasma plume arrives at the Mach probe location at the midplane at about $t=30 \mu \mathrm{s}$, where $t=0$ corresponds to initial plasma breakdown in the gun. The transit time for the plasma plume to reach the Mach probe at the midplane (about $0.5 \mathrm{~m}$ ) is about $10 \mu \mathrm{s}$. We typically observe high frequency fluctuations as the plasma evolves in the wind tunnel $(t=30-50 \mu \mathrm{s})$ but late in time the fluctuations subside. In Fig. 4, we show measurements of ion temperature $T_{i}$ determined with ion Doppler spectroscopy ${ }^{17}$ and electron temperature $T_{e}$ determined with an impurity line ratio technique using vacuum ultraviolet spectroscopy. ${ }^{18}$ Note that early in time, $T_{i} \geq T_{e}$ but the temperatures tend to equilibrate around $50 \mu \mathrm{s}$. Both temperatures are line-averaged measurements along a chord passing below the axis of the machine. Using Eq. (2), we can calculate the local sound speed as a function of time (Fig. 5). Note that the sound speed is highest when the sum of $T_{i}+T_{e}$ peaks $(30-40 \mu \mathrm{s})$. We find the local sound speed in the initial plasma plume to be $c_{s}=52.5 \pm 4.4 \mathrm{~km} / \mathrm{s}$.

To absolutely calibrate the Mach probe in the SSX wind tunnel, a linear magnetic probe array was constructed and placed along the wind tunnel axis. The probe separation was $4.8 \mathrm{~cm}$ and 14 two-axis probes were used. $B_{x}$ and $B_{y}$ are measured and we report the magnitude $|\mathbf{B}|$. The axial field $B_{z}$ should be small on axis. Under the MHD assumption of frozen-in flux, the plasma and magnetic field should flow together down the wind tunnel. Time-of-flight analysis is used to determine the initial flow velocity of the plasma plume. In Fig. 6, we plot $|\mathbf{B}|$ for probes 5, 8, and 12 on a single discharge. The boxes indicate the arrival times of the magnetized plasma plume using an automated peak detection algorithm. Finally, in Fig. 7 we plot the mean arrival times of $|\mathbf{B}|$ at each probe for 40 discharges (20 each of RH and LH plumes). The error bars are the standard errors for arrival times at each magnetic probe location. For the purposes of calibration, we use the slope of a linear regression of the mean arrival times for each probe. Aggregating over 40 plasma shots, the data from the linear array of magnetic probes give $v_{\text {plume }}=55.1 \pm 6.4 \mathrm{~km} / \mathrm{s}$ at about $32 \mu \mathrm{s}$. Evidently, the Mach number of the initial flow is about unity, using the sound speed determined above.

\section{B. Analysis}

Because of the particular SSX plasma parameters and the cylindrical geometry of the Mach probe, there are no calibration constants readily available in literature for the current configuration. Furthermore, from ion temperature measurements (Fig. 4) and magnetic field measurements (Fig. 6), we can calculate the proton gyro-radius. For $T_{i}=20 \mathrm{eV}$ and $|\mathbf{B}|=0.1 \mathrm{~T}$, we find a proton gyro-radius of about $0.5 \mathrm{~cm}$, while the radius of our probe is $0.6 \mathrm{~cm}$. This defines for us the weakly magnetized regime. In addition, since the ion-ion collision frequency for $20 \mathrm{eV}$ is about $0.5 \mathrm{MHz}$ while the proton gyro frequency at $0.1 \mathrm{~T}$ is about $1.5 \mathrm{MHz}$, we conclude that a typical proton executes only a few orbits before suffering a collision. So, in this sense too, our ions are weakly magnetized. Though Patacchini's updated numerical model provides theoretical guidance for probes operating under similar, weakly magnetized regimes, there are no experimentally verified calibration parameters for probe operation. 
(a)
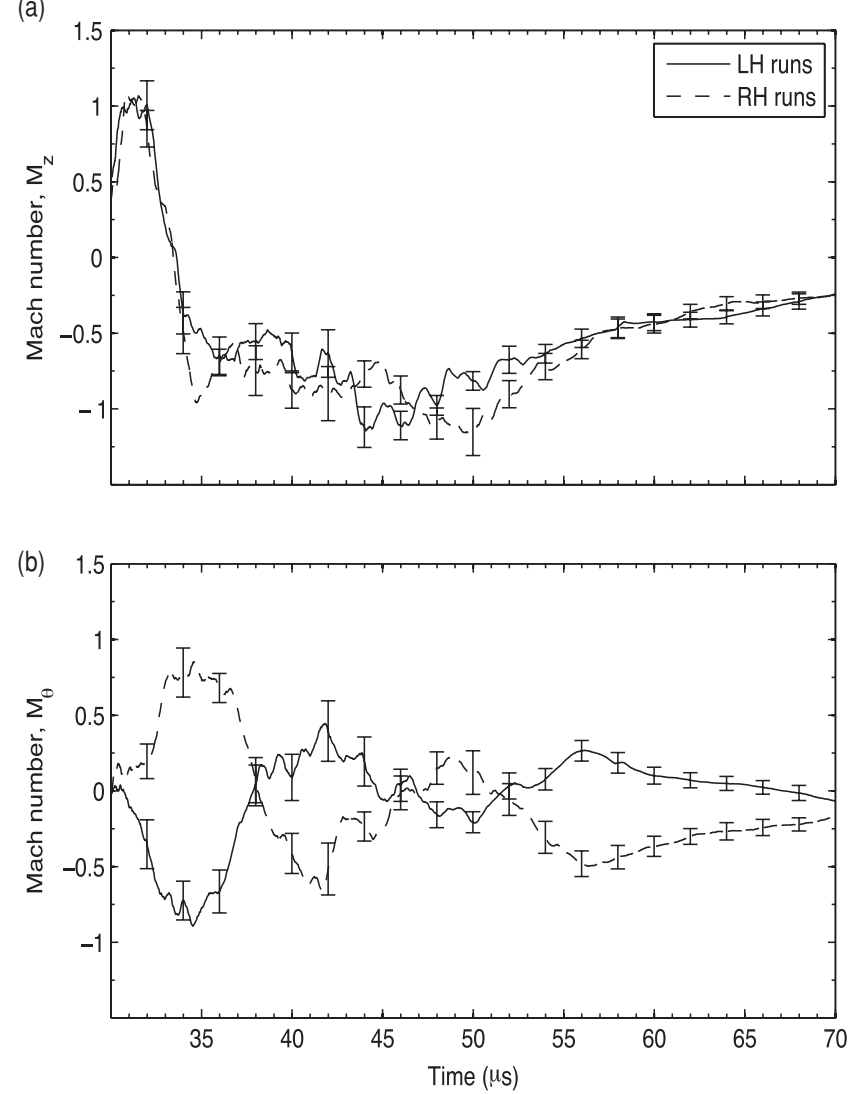

FIG. 8. Mach number in the (a) $z$-direction and (b) $\theta$-direction for LH and RH shots aggregated over 20 discharges in each orientation. Peak initial $M_{z}$ flows are observed at approximately $32 \mu \mathrm{s}$. Note that very little azimuthal flow is observed in the initial plume.

Using data such as depicted in Fig. 3, we can apply Eq. (1) to calculate the Mach number of the flow. Since we have directly measured the initial flow speed of the plume and the local sound speed as the plume arrives at the midplane, we can perform a fit to determine the constant $K$ for the SSX Mach probe. In Fig. 8(a), we show the mean axial flow speed as a function of time for both $\mathrm{LH}$ and $\mathrm{RH}$ plasma plumes. The best fit for the calibration constant is: $K=2.0 \pm 0.5$. Figure 8(b) depicts the azimuthal Mach number as a function of time for the aggregate set of discharges. Note that the $\mathrm{RH}$ plumes and LH plumes have opposite flow patterns as a function of time, while the axial flow patterns are identical for $\mathrm{RH}$ and LH. In addition, note that there is very little azimuthal flow initially.

As noted above, Mach probes report only the flow speed normalized to the local sound speed. We can extract the absolute flow speed by multiplying the results in Fig. 8 by the sound speed plot in Fig. 5. In Figs. 9(a) and 9(b), we present that result.

\section{PARTICLE SIMULATIONS}

A particle pushing code (PPC) was used to simulate ion flux to the collector faces of the SSX Mach probe. PPC is a modification and extension of the code designed for tracing charged particle orbits in analytically specified electromag- netic fields. ${ }^{19}$ The resulting simulated plasma is collisionless and the effects of self-consistent fields are ignored. The data collected from the simulations were used to get a theoretical value for the calibration constant $K$ under the simplified version of the SSX wind tunnel plasmas modeled by PPC.

A cylindrical test chamber with radius $R=0.08 \mathrm{~m}$ and length $0.32 \mathrm{~m}$ housing a Mach probe with the same dimensions as the SSX probe was simulated. The coordinate axes of the system are situated such that the length of the cylinder (and the direction of the particle drifts) is along the $z$-axis, with the Mach probe protruding inward along the $y$-axis. A constant $0.1 \mathrm{~T}$ magnetic field directed in the $+z$-direction was used as a simple model for local fields. To account for both the dynamic nature of the magnetic fields and the weakly collisional nature of the plasma in SSX, a simulation time of two microseconds was used; this run-time is approximately equal to the average time between collisions of protons in SSX plasmas.

The initial position of each particle was chosen at random from a uniform distribution within the cylindrical test chamber. The initial velocity of each particle was drawn from a Maxwellian distribution of $T=20 \mathrm{eV}$ (typical of SSX plasmas), with varying superimposed drifts in the $+z$-direction used to model different net drift velocities of the plasma. The drift speeds spanned the typical range for SSX plasma, from $M=0$ to $M=2$. For each run, the number of particle hits on the sensors of the probe were recorded, with the number of hits on a particular sensor being considered proportional to the total current $J$ incident on that sensor. Each run used 50 $\times 10^{6}$ particles. Sheath effects were ignored.

The results of these simulations are shown in Fig. 10. We plot $\ln \left(J_{\text {up }} / J_{\text {down }}\right)$ which we assume to be proportional
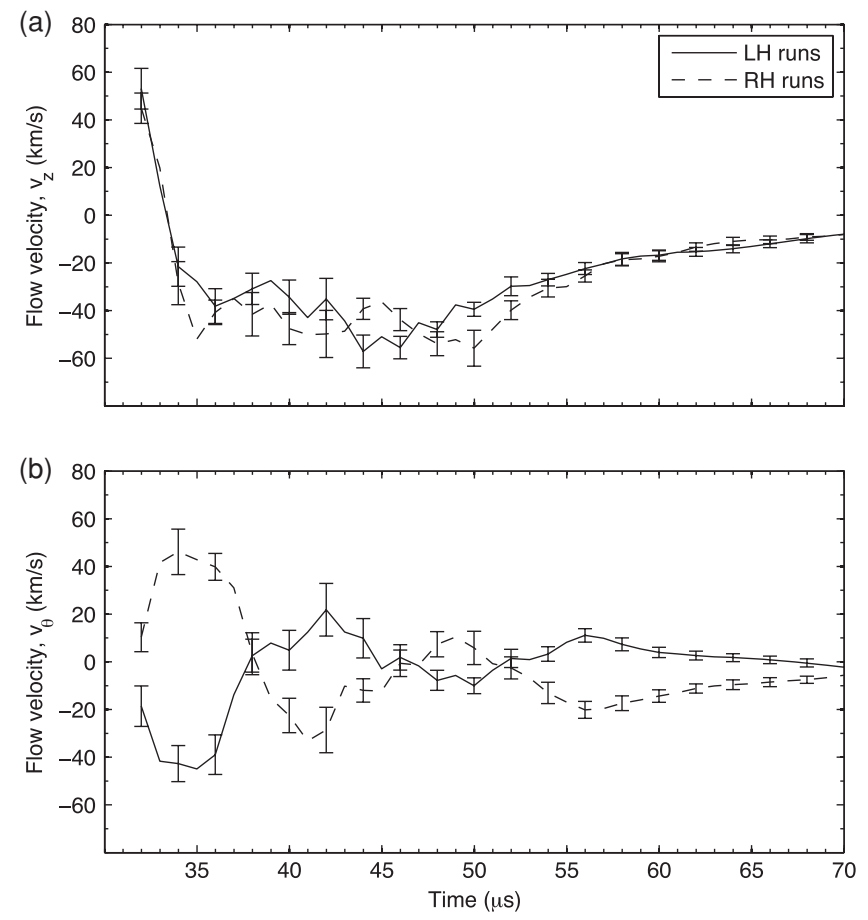

FIG. 9. Absolute flow velocities in the (a) $z$-direction and (b) $\theta$-direction. Data included for LH and RH shots aggregated over 20 discharges. 


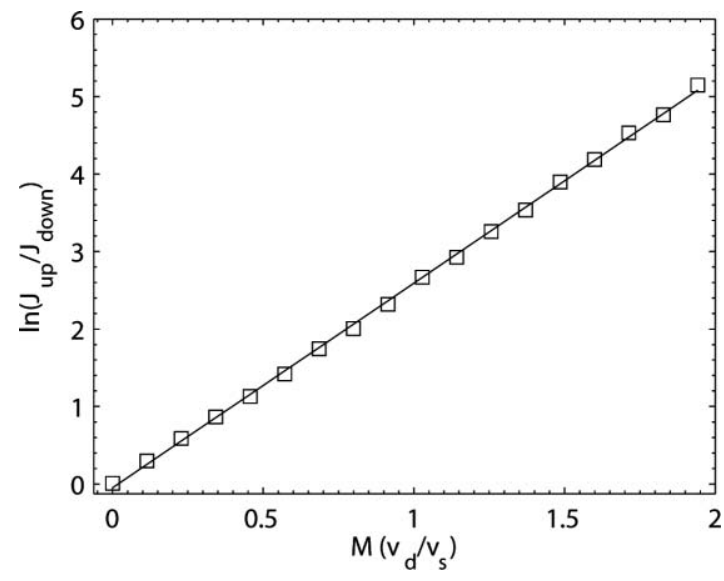

FIG. 10. Simulation plot of z-directed Mach number (using the expression $\left.\left(M_{1}+M_{2}\right) / 2 \cos 30^{\circ}\right)$ versus the exact input Mach number (defined by $\left.v_{d} / v_{t h}\right)$. The error bars are smaller than the resolution of the graph. A linear least-squares fit to the data gives an implied calibration constant of $K=2.61 \pm 0.01$.

to the Mach number $M$ with proportionality constant $K$. Sensors typically registered between 100 and 1000 particle hits each. The plot shows the recorded z-directed Mach number using the expression $\left(M_{1}+M_{2}\right) / 2 \cos 30^{\circ}$ versus the exact input Mach number (defined by $v_{d} / v_{t h}$ ). The error bars are smaller than data markers on the graph. A linear leastsquares fit to the data gives an implied calibration constant of $K=2.61 \pm 0.01$.

\section{DISCUSSION AND SUMMARY}

A simple six-faced Mach probe is described and a calibration procedure is presented. From time-of-flight measurements in the SSX wind tunnel, with flow speeds of about $50 \mathrm{~km} / \mathrm{s}$, absolute calibration of the probe to the functional form $J_{\text {up }} / J_{\text {down }}=e^{K M}$ gives $K=2.0 \pm 0.5$. The SSX regime is neither in the un-magnetized nor fully magnetized limit so we employed a particle simulation to corroborate our measurement. Using $50 \times 10^{6}$ particles drawn from a Maxwellian distribution of $T=20 \mathrm{eV}$ at a variety of drift speeds relative to the thermal speed gives a calibration constant of $K=2.61 \pm 0.01$ with a uniform magnetic field.

We note that ion magnetization can be expected to influence the details of the probe model. In particular, we note that in both analytical models and simulations wherein ions are strongly magnetized $\rho_{i} / r_{\text {probe }} \ll 1$, the calibration constant $K$ tends to lower values. (For example, in the magnetized regime, Hutchinson ${ }^{20}$ has derived $K$ $=1 /\left(0.43 \sqrt{1+T_{i} / T_{e}}\right)=1.2$ for typical SSX parameters. $)$ Similarly, directionality of the magnetic field with respect to the drift velocity and magnetic field gradients could affect interpretation of the probe data. More simulation studies with more realistic magnetic field geometries are planned in the near future.

\section{ACKNOWLEDGMENTS}

This work was supported by grants from the Department of Energy, the National Science Foundation (CMSO), and the Office of Naval Research. The authors gratefully acknowledge the assistance of S. Palmer, J. Haldeman, A. H. Glasser, M. Korein, B. Gerber-Siff, K. Labe, and D. Weinhold.

${ }^{1}$ M. Hudis and L. M. Lidsky, J. Appl. Phys. 41, 5011 (1970).

${ }^{2}$ I. H. Hutchinson, Phys. Plasmas 9, 1832 (2002).

${ }^{3}$ S. C. Hsu, T. A. Carter, G. Fiksel, H. Ji, R. M. Kulsrud, and M. Yamada, Phys. Plasmas 8, 1916 (2001).

${ }^{4}$ L. Oksuz, M. A. Khedr, and N. Hershkowitz, Phys. Plasmas 8, 1729 (2001).

${ }^{5}$ B. J. Peterson, J. N. Talmadge, D. T. Anderson, F. S.B. Anderson, and J. L. Shohet, Rev. Sci. Instrum. 65, 2599 (1994).

${ }^{6}$ W. M. Solomon and M. G. Shats, Rev. Sci. Instrum. 72, 449 (2001).

${ }^{7}$ J. P. Gunn, C. Boucher, P. Devynck, I. Duran, K. Dyabilin, J. Horacek, M. Hron, J. Stockel, G. V. Oost, H. V. Goubergen, and F. Zacek, Phys. Plasmas 8, 1995 (2001).

${ }^{8}$ C. S. MacLatchy, C. Boucher, D. A. Poirier, and J. Gunn, Rev. Sci. Instrum. 63, 3923 (1992).

${ }^{9}$ M. R. Brown, Phys. Plasmas 6, 1717 (1999).

${ }^{10}$ K.-S. Chung, Jpn. J. Appl. Phys. 45, 7914 (2006).

${ }^{11}$ H. M. Mott-Smith and I. Langmuir, Phys. Rev. 28, 727 (1926).

${ }^{12}$ L. Oksuz and N. Hershkowitz, Plasma Sources Sci. Technol. 13, 263 (2004).

${ }^{13}$ I. H. Hutchinson, Plasma Phys. Controlled Fusion 44, 1953 (2002).

${ }^{14}$ L. Patacchini and I. H. Hutchinson, Plasma Phys. Controlled Fusion 52, 035005 (2010),

${ }^{15}$ T. Gray, M. R. Brown, C. D. Cothran, G. Marklin, and M. J. Schaffer, Phys. Plasmas 17, 032510 (2010).

${ }^{16}$ T. Gray and M. R. Brown, "Selective Decay in a Quasi-Infinite Cylinder," Phys. Rev. Lett. (submitted).

${ }^{17}$ C. D. Cothran, J. Fung, M. R. Brown, and M. J. Schaffer, Rev. Sci. Instrum. 77, 063504 (2006).

${ }^{18}$ V. H. Chaplin, M. R. Brown, D. H. Cohen, T. Gray, and C. D. Cothran, Phys. Plasmas 16, 042505 (2009).

${ }^{19}$ A. H. Glasser and S. A. Cohen, Phys. Plasmas 9, 2093 (2002).

${ }^{20}$ I. Hutchinson, Phys. Rev. A 37, 4358 (1988). 